\title{
Pattern of psychotropic prescription in a tertiary care hospital of Bangladesh: a cross-sectional analysis of prescription photos
}

\author{
Hussain $\mathrm{F}^{1}$, Alam $\mathrm{M}^{1}$, Mali $\mathrm{B}^{2}$, Akter $\mathrm{H}^{2}$ and Arafat $\mathrm{SMY}^{3 *}$ \\ ${ }^{1}$ Department of Pharmacy, Noakhali Science and Technology University, Noakhali, Bangladesh \\ ${ }^{2}$ Bangabandhu Sheikh Mujib Medical University, Dhaka, Bangladesh \\ ${ }^{3}$ Department of Psychiatry, Bangabandhu Sheikh Mujib Medical University, Dhaka, Bangladesh
}

\section{Introduction}

Mental disorders account for a growing proportion of the worldwide burden of diseases. In 2013, mental disorders accounted for $14 \%$ of the worldwide burden of diseases [1]. The burden of mental health disorders in Bangladesh is yet to be measured precisely as done in the developed countries. A recent systemic review reported the prevalence of mental disorders varied from 6.5 to $31.0 \%$ among adults and 3.4 to $22.9 \%$ among children in the country [2]. As a part of holistic biopsychosocial approach, pharmacotherapy is an important part of care for mentally ill patients [3]. But, pattern of prescriptions varies based on the different geographical areas, patient characteristics, type of disorder, culture, environment, socioeconomic status, availability drugs and prescribing habit of physicians [4]. Drug surveillance studies seek to monitor and evaluate the prescribing patterns to make the care evidence based, rational and cost-effective [4]. Moreover, describing the pattern also helps in identifying the factors responsible for irrational practice of polypharmacy as well as problems associated with it [5]. We aimed to see prescription pattern of psychotropics in a tertiary care specialized psychiatric hospital at out patient services setting of Bangladesh.

\section{Methods}

This hospital-based descriptive cross-sectional study was conducted within the period of January to March 2018 at the outpatient department (OPD) of a tertiary care specialized mental health hospital of Dhaka, Bangladesh. Convenient sampling was used to collect data from 709 patients visited at the study duration by capturing the photos of the prescriptions with a smart phone. All prescriptions having at least one psychotropic drug were included. Prescriptions in which hand-writing could not be comprehensible, or advised for admission, and referred to other hospitals were excluded. Permission was taken from the ethical review committee of the department of Pharmacy of Noakhali Science and Technology University and confidentiality of data was maintained adequately. Data were analyzed using the Statistical Package for Social Science (SPSS) version 20 for Windows and Microsoft Excel version 2010. The psychotropic drugs included for analysis were antipsychotics, antidepressants, mood stabilizers anti-cholinergics and sedativehypnotics.

\section{Results}

Among the 709 photos of outpatient services prescriptions $454(64 \%)$ were male and $255(36 \%)$ were females. The mean age of the respondents was $29.63(\mathrm{SD} \pm 11.43)$ years, ranging from 4 to 70 years. The mean of total number of drugs in a prescription was 3.42 (SD \pm 0.64 ). About $75 \%$ of the prescriptions had anti-psychotics, $42 \%$ had anti-depressants, $17 \%$ had long acting injections (LAIs) and $63 \%$ of the prescriptions had combination of drugs (Table 1). Among the antipsychotics, olanzapine was found in about $14 \%$ of the prescriptions, followed by risperidone and others. Mirtazapine was most commonly found among the anti-depressants, clonazepam was most commonly hypnotics, fluphenazine was most common LAIs, valproate preparation was most common mood stabilizer and procyclidine was most common anti-cholinergic. Majority of prescriptions contained single drug from the specific groups. However, combinations were also found as there were even three anti-psychotics in the same prescription. The category other contained vitamins and minerals, food supplements, anti-ulcerants, hormone products and drugs others than psychotropics (Table 2).

\section{Discussion}

We aimed to see prescription pattern of psychotropics of at OPD of a tertiary care specialized mental health care center in Dhaka, Bangladesh. In the current study, anti-psychotics were most commonly prescribed drugs and about $75 \%$ of the prescriptions contained antipsychotics. The mean of total number of drugs including other drugs in a prescription was $3.42(\mathrm{SD} \pm 0.64)$. Nearly similar number of psychotropics (3.39 \pm 1.67$)$ were found in a study by Mukherjee et al., in 2014 [5]. Another study revealed the average drug was $2.51 \pm 0.75$ [4]. Similar pattern was found in a study conducted in India [6]. About $64 \%$ of the prescriptions contained combination of psychotropics in the OPD setting. In a study in India revealed that, about $77 \%$ of the

*Correspondence to: SM Yasir Arafat, Resident, Department of Psychiatry, Bangabandhu Sheikh Mujib Medical University, Dhaka, Bangladesh, Tel: +8801713272917; E-mail: arafatdmc62@gmail.com

Received: January 18, 2018; Accepted: January 27, 2018; Published: January 30, 2018 
Table 1. Distribution of psychotropics ( $\mathrm{n}=709)$

\begin{tabular}{|c|c|c|}
\hline Psychotropics & Frequency & Percent \\
\hline Anti-psychotics & 533 & 75.17 \\
\hline Anti-cholinergic (mostly procyclidine) & 411 & 57.96 \\
\hline Anti-depressants & 295 & 41.60 \\
\hline Sedatives & 216 & 30.46 \\
\hline Mood stabilizers & 125 & 17.63 \\
\hline Anti-dementics & 57 & 8.03 \\
\hline Long acting injection (Fluphenazine) & 121 & 17.06 \\
\hline Others & 289 & 40.76 \\
\hline Combination & 448 & 63.20 \\
\hline \multicolumn{3}{|l|}{ Anti-psychotics } \\
\hline Olanzapine & 97 & 13.74 \\
\hline Risperidone & 75 & 10.51 \\
\hline Trifluoperazine & 34 & 4.85 \\
\hline Quetiapine & 34 & 4.85 \\
\hline Fluphenazine (LAI) & 11 & 1.62 \\
\hline Chlorpromazine & 11 & 1.62 \\
\hline Haloperidol & 6 & 0.81 \\
\hline Aripiprazole & 6 & 0.81 \\
\hline Clozapine & 6 & 0.81 \\
\hline Combined & 252 & 33.57 \\
\hline Total & 533 & 75.18 \\
\hline \multicolumn{3}{|l|}{ Anti-depressants } \\
\hline Mirtazapine & 89 & 12.56 \\
\hline Sertraline & 56 & 7.85 \\
\hline Escitalopram & 33 & 4.71 \\
\hline Fluoxetine & 17 & 2.36 \\
\hline Clomipramine & 17 & 2.36 \\
\hline Amitriptyline & 6 & 0.79 \\
\hline Venlafaxine & 6 & 0.79 \\
\hline Combination & 72 & 10.21 \\
\hline Total & 295 & 41.61 \\
\hline \multicolumn{3}{|l|}{ Sedatives } \\
\hline Clonazepam & 168 & 23.70 \\
\hline Diazepam & 30 & 4.23 \\
\hline Midazolam & 6 & 0.85 \\
\hline Bromazepam & 6 & 0.85 \\
\hline Combination & 6 & 0.85 \\
\hline Total & 216 & 30.47 \\
\hline
\end{tabular}

Table 2. Distribution of prescription of multiple drugs of same group

\begin{tabular}{|c|c|c|c|c|c|c|}
\hline Number of Drugs & \multicolumn{2}{|c|}{ Antipsychotic } & \multicolumn{2}{c|}{ Antidepressants } & \multicolumn{2}{c|}{ Mood stabilizers } \\
\hline & $\mathrm{N}$ & $\%$ & $\mathrm{~N}$ & $\%$ & $\mathrm{~N}$ & $\%$ \\
\hline 1 & 250 & 35.2 & 224 & 31.59 & 95 & 13.40 \\
\hline 2 & 238 & 33.6 & 71 & 10.02 & 30 & 4.23 \\
\hline 3 & 45 & 6.4 & & & & \\
\hline
\end{tabular}

patients received polypharmacy [6]. Another study in India revealed $85 \%$ of the patients had found to get polypharmacy [5]. Among the anti-psychotics, olanzapine was most common which was similar in Ghana where olanzapine was the commonly prescribed psychotropic medication [1]. Other studies in India also found olanzapine as the commonest anti-psychotic [4,7]. Mirtazapine was commonest antidepressant and clonazepam was comment hypnotics. Rode et al., found escitalopram, and clonazepam as the commonest ones respectively [4].

The study revealed about $17 \%$ of the prescriptions had LAIs whereas another study among 120 schizophrenia patients revealed about $57 \%$ of the patients had history of receiving LAIs [8]. The variations can be explained by the different distribution of patients in the two different settings. Current study included prescriptions of patients without any diagnosis, but the mentioned study included only schizophrenic patients. However, Ashong et al., didn't found any evidence of LAIs among schizophrenic patient because of cost, insurance coverage, treatment setting, negative attitudes of healthcare professionals and patients, fear, and pain on injection [1]. But, in India perceived expenses of LAIs is an important cause of low LAI using [810]. Mukherjee et al., found only $4.42 \%$ respondents were found to get parenteral preparations [5].

Though the study can provide an initial idea of further studies, but with this cross sectional, single centered, generalization of the study findings would be difficult. Moreover, the study didn't give any rationale of the prescribing the psychotropics and diagnoses were not assessed. Further larger scale studies considering the diagnosis and rationale of prescription can be considered.

\section{Conclusions}

Two-third of the patients of OPD getting anti-psychotics, olanzapine was mostly prescribed anti-psychotic, mirtazapine was most commonly anti-depressant, clonazepam was most commonly prescribed hypnotic. More than 6 out of 10 patients were prescribed combination of psychotropics.

\section{Funding}

Self-funded.

\section{Conflict of interest}

None.

\section{References}

1. Ashong S, Kretchy IA, Afrane B, Aikins A-G (2018) Patterns of Prescription of Psychotropic Medications and Their Adherence among Patients with Schizophrenia in Two Psychiatric Hospitals in Accra, Ghana: A Cross-Sectional Survey. Psychiatry $J$ $10.1155 / 2018 / 9850594$.

2. Hossain MD, Ahmed HU, Chowdhury WA, Niessen LW, Alam DS (2014) Mental disorders in Bangladesh: a systematic review. BMC Psych 14: 216.

3. Engel GL (1980) The clinical application of the biopsychosocial model. Am J Psychiatry 137: 535-544. [Crossref]

4. Rode SB, Ajagallay RK, Salankar HV, Sinha U (2014) A study on drug prescribing pattern in psychiatry out-patient department from a tertiary care teaching hospital. Int $J$ Basic Clin Pharmacol 3: 517-22.

5. Mukherjee S, Sen S, Chatterjee SS, Biswas A, Sinha S, et al. (2014) Prescribing Pattern of Psychotropic Medications in Psychiatry Outpatients at a Tertiary Care Teaching Hospital in India: A Prospective Cross-sectional Study. Int J Hosp Res 3: 113-122.

6. Sarkar P, Chakraborty K, Misra A, Shukla R, Swain SP (2013) Pattern of psychotropic prescription in a tertiary care center: A critical analysis. Indian J Pharmacol 45: 270273. [Crossref]

7. Venkataraman R, Rayamajhi M, Islam S, Nanjunda N (2018) Prescribing Pattern of Psychotropic Agents in Rural Tertiary Care Teaching Hospital. Asian J Pharm Clin Res 11: 155-62

8. Arafat SMY, Mali B, Akter H (2018) Proportion and reasons for medication noncompliance among schizophrenics: A cross-sectional observation in a tertiary care hospital of Bangladesh. Asian J Psychiatr 35: 52-54. [Crossref]

9. Gundugurti PR, Nagpal R, Sheth A, Narang P, Gawande S, et al. (2017) Effects of oral versus long-acting antipsychotics on social functioning: a psychiatrists' survey in India Asian J Psychiatr 30: 88-93. [Crossref]

10. Sreeraj VS, Shivakumar V, Rao NP, Venkatasubramanian G (2017) A critical appraisal of long acting injectable antipsychotics: translating research to clinics. Asian $J$ Psychiatr 28: 57-64. [Crossref] 
Hussain F (2018) Pattern of psychotropic prescription in a tertiary care hospital of Bangladesh: a cross-sectional analysis of prescription photos

Copyright: $(02018$ Hussain F. This is an open-access article distributed under the terms of the Creative Commons Attribution License, which permits unrestricted use, distribution, and reproduction in any medium, provided the original author and source are credited. 